Dataset Paper

\title{
Evaluating Wheat Microsatellite Markers for the Use in Genetic Analysis of Thinopyrum, Dasypyrum, and Pseudoroegneria Species
}

\author{
Pavel Yu. Kroupin, Mikhail G. Divashuk, Igor A. Fesenko, and Gennady I. Karlov \\ Centre for Molecular Biotechnology, Russian State Agrarian University-Moscow Timiryazev Agricultural Academy, \\ Timiryazevskaya Street 49, Moscow 127550, Russia
}

Correspondence should be addressed to Pavel Yu. Kroupin; pavelkroupin@gmail.com

Received 25 May 2012; Accepted 18 July 2012

Academic Editors: M. Chen, S. A. Mohammadi, and G. Sun

Copyright (C) 2013 Pavel Yu. Kroupin et al. This is an open access article distributed under the Creative Commons Attribution License, which permits unrestricted use, distribution, and reproduction in any medium, provided the original work is properly cited.

\begin{abstract}
A set of 42 SSRs of wheat were evaluated for their cross-amplification on the DNA of Thinopyrum ponticum, Thinopyrum intermedium, Thinopyrum elongatum, Thinopyrum bessarabicum, Pseudoroegneria stipifolia, and Dasypyrum villosum. The number of the wheat SSR markers that amplified DNA fragments with determined size for Th. ponticum was 33 (78.6\%); for Th. intermedium, 28 (66.7\%); for Th. elongatum, 24 (57.1\%); for Th. bessarabicum, 24 (57.1\%); for P. stipifolia, 26 (69.1\%); and for D. villosum, 29 (69.0\%). Twenty-four primer pairs of wheat SSR markers were successfully amplified from all investigated species. The dataset can be used for phylogenetic studies of wild relatives of wheat, for the estimation of their diversity, and for the introgression of agronomically valuable genes into wheat genome.
\end{abstract}

\section{Introduction}

Wheat is a major food source for most world population. However, its cultivation is strictly limited by such challenges as pests, diseases, droughts, extreme temperatures, and others. In order to manage sustainable wheat production, it is necessary to develop new cultivars of wheat resistant and tolerant to adverse environmental factors. The genetic diversity of wheat is restricted due to the bottleneck effect which occurred during domestication and, on the other hand, due to the limited diversity of wheat germplasm used in the breeding programs $[1,2]$. The genetic variability of cultivated wheat can be improved by wide hybridization involving wild relative species. One of the important gene pools is different species of Thinopyrum, Dasypyrum, and Pseudoroegneria genera $[3,4]$. These genetic resources can be efficiently used in wheat breeding by application of molecular microsatellite markers. Microsatellites also known as Simple Sequence Repeats (SSRs) are tandem repeats of two to six nucleotide DNA motifs randomly distributed all over genome. They have been successfully used as DNA markers to estimate genetic diversity, genome mapping, and marker-assisted selection for agronomically important traits [5]. SSR markers are a powerful tool for the investigation of cereal genomes because of the high level of polymorphism they reveal [6]. In addition, SSRs can be used in wide hybridization of wheat to monitor and map desirable alien genes in segregating populations as they are locus specific and inherited in codominant manner [7].

A large number of the primer sequences of wheat SSRs are publicly available. However, the development of new SSR markers de novo for wild relatives of wheat is a time- and costconsuming process. The flanking regions of microsatellites show sufficient homology between closely related species. Thus, the primers of wheat SSR markers can be used in the analysis of other Poaceae species. This is called "transferability" and it has been successfully used for cultivated (barley, oats, and rye) [8] and wild (Elymus, Aegilops) species [9, 10]. The goal of our study was to test a set of 42 microsatellite markers of wheat for their ability to cross-amplify DNA from the accessions of Th. intermedium, Th. elongatum, $T h$. ponticum, Th. bessarabicum, P. stipifolia, and D. villosum. 


\section{Methodology}

To get the plant materials, the following accessions were ordered from Germplasm Research International Network: Th. ponticum (PI 547312), Th. intermedium (PI 401200), Th. bessarabicum (PI 531711), Th. elongatum (PI 401007) P. stipifolia (W6 21759), and D. villosum (W6 21717). Two plants of each accession were analyzed. DNA was extracted from etiolated seedling by the standard CTAB method.

Polymerase chain reaction (PCR) and fragment analysis were performed as follows. PCR was performed in final volume of $25 \mu \mathrm{L}$ in thermal cycler using the conditions recommended at GrainGenes 2.0 [11] according to the protocol by Schuelke [12]. The first round of PCR was conducted using reverse $(0.16 \mathrm{pmol} / \mu \mathrm{L})$ and forward primer with M13 tail $(0.04 \mathrm{pmol} / \mu \mathrm{L})$. During the second round, the fluorescent-labeled M13 primer was incorporated into the PCR product at annealing temperature $53^{\circ} \mathrm{C}$. Fragment analysis of PCR product was carried out using ABI 3130xl Genetic Analyzer (Applied Biosystems, Foster City, CA, USA) with GeneScan 500 LIZ size standard according to the manufacturer's recommendations. The sizes of fragments were analyzed using Peak Scanner Software v1.0 (Applied Biosystems, Foster City, CA, USA).

\section{Dataset Description}

The dataset associated with this Dataset Paper consists of one item which is described as follows.

Dataset Item 1 (Table). Size of the microsatellite fragments (base pair (bp)) amplified on DNA of Th. intermedium, Th. elongatum, $P$. stipifolia, Th. bessarabicum, Th. ponticum, and D. villosum. A total of 42 SSR markers of wheat were evaluated on their cross-amplification on DNA of Th. intermedium, Th. elongatum, Th. ponticum, Th. bessarabicum, P. stipifolia, and D. villosum. For each accession, two plants were analyzed. The sizes of the peaks are obtained by means of the fragment analysis. The number of the wheat SSR markers that amplified DNA fragments with determined size for Th. ponticum was 33 (78.6\%); for Th. intermedium, 28 (66.7\%); for Th. elongatum, 24 (57.1\%); for Th. bessarabicum, 24 (57.1\%); for P. stipifolia, 26 (69.1\%); and for D. villosum, 29 (69.0\%). Thus, the transferability of the SSR markers to the genomes of the investigated species is comparable to that of oat, rye [8], Aegilops [9, 13], Elymus, and Pseudoroegneria [10]. Twentyfour primer pairs of wheat SSR markers were successfully amplified from all investigated species. The absence of amplification may result from either null allele in a locus or significant differences between wheat and studied species in the flanking regions where primers should anneal [14]. The transferred SSRs can be linked to useful genes or QTLs as among selected markers there are some previously used for the mapping and detection of resistance genes of $T h$. intermedium (Xwmc221, Xwmc121, and Xcfd68) [15] and Th. elongatum (Xgwm325, Xgwm179, and Xgwm335) [16, 17] and others are linked to QTLs of valuable traits on different wheat chromosomes [18]. The size shown in the table is the sum of the real size of a fragment and 25 bp of M13 tail of forward primer. The localization on wheat chromosomes is shown according to Graingenes 2.0 database [10]. If no peaks are detected, they are shown as "0" size. Where the variants between plant genotypes are observed, they are shown through slash " $/$ ". If a group of peaks is found for one plant and it differs from the group of another plant, the sizes of peaks are shown in brackets. Cases when it is difficult to determine major peaks among multiple peaks are designated "ND" (not determined).

\section{Column 1: SSR Marker}

Column 2: Localization in Wheat Chromosome

Column 3: Size of the Amplified Fragments of Th. intermedium (bp)

Column 4: Size of the Amplified Fragments of Th. elongatum (bp)

Column 5: Size of the Amplified Fragments of P. stipifolia (bp)

Column 6: Size of the Amplified Fragments of Th. bessarabicum (bp)

Column 7: Size of the Amplified Fragments of Th. ponticum (bp)

Column 8: Size of the Amplified Fragments of D. villosum (bp)

\section{Concluding Remarks}

In conclusion, it has been shown that most of 42 SSRs are applicable for the investigation of genomes of Th. ponticum, Th. intermedium, Th. elongatum, Th. bessarabicum, P. stipifolia, and D. villosum. The polymorphism in SSR fragment size between different analyzed species has been shown. The transferred SSR markers can be used in assaying the genetic variability of these wild species and establishment of phylogenetic relationships between them. In addition, they can be used in wide hybridization of wheat to monitor and map alien introgressions.

\section{Dataset Availability}

The dataset associated with this Dataset Paper is dedicated to the public domain using the CCO waiver and is available at http://dx.doi.org/10.7167/2013/949637/dataset.

\section{Acknowledgment}

This work was supported by the Federal Target Program "Scientific and Scientific-Educational Professionals of Innovative Russia” for 2009-2013, state contract P1048.

\section{References}

[1] S. P. Martynov, T. V. Dobrotvorskaya, and V. A. Pukhalskiy, "Dynamics of genetic diversity in winter common wheat Triticum aestivum L. cultivars released in Russia from 1929 to 2005," Russian Journal of Genetics, vol. 42, no. 10, pp. 1137-1147, 2006. 
[2] J. Dubcovsky and J. Dvorak, "Genome plasticity a key factor in the success of polyploid wheat under domestication," Science, vol. 316, no. 5833, pp. 1862-1866, 2007.

[3] A. Gradzielewska, "The genus Dasypyrum-part 2. Dasypyrum villosum - a wild species used in wheat improvement," Euphytica, vol. 152, no. 3, pp. 441-454, 2006.

[4] H. Li and X. Wang, "Thinopyrum ponticum and Th. intermedium: the promising source of resistance to fungal and viral diseases of wheat," Journal of Genetics and Genomics, vol. 36, no. 9, pp. 557-565, 2009.

[5] R. K. Kalia, M. K. Rai, S. Kalia, R. Singh, and A. K. Dhawan, "Microsatellite markers: an overview of the recent progress in plants," Euphytica, vol. 177, no. 3, pp. 309-334, 2011.

[6] M. S. Röder, V. Korzun, K. Wendehake et al., "A microsatellite map of wheat," Genetics, vol. 149, no. 4, pp. 2007-2023, 1998.

[7] X. Shen, L. Kong, and H. Ohm, "Fusarium head blight resistance in hexaploid wheat (Triticum aestivum)-Lophopyrum genetic lines and tagging of the alien chromatin by PCR markers," Theoretical and Applied Genetics, vol. 108, no. 5, pp. 808-813, 2004.

[8] A. Yildirim, N. Kandemir, O. A. Sönmezoğlu, and T. E. Güleç, "Transferability of microsatellite markers among cool season cereals," Biotechnology and Biotechnological Equipment, vol. 23, no. 3, pp. 1299-1302, 2009.

[9] L. Y. Zhang, M. Bernard, P. Leroy, C. Feuillet, and P. Sourdille, "High transferability of bread wheat EST-derived SSRs to other cereals," Theoretical and Applied Genetics, vol. 111, no. 4, pp. 677-687, 2005.

[10] D. MacRitchie and G. Sun, "Evaluating the potential of barley and wheat microsatellite markers or genetic analysis of Elymus trachycaulus complex species," Theoretical and Applied Genetics, vol. 108, no. 4, pp. 720-724, 2004.

[11] GrainGenes 2.0, http://wheat.pw.usda.gov/GG2/index.shtml .

[12] M. Schuelke, "An economic method for the fluorescent labeling of PCR fragments," Nature Biotechnology, vol. 18, pp. 233-234, 2000.

[13] I. G. Adonina, E. A. Salina, E. G. Pestsova, and M. S. Röder, "Transferability of wheat microsatellites to diploid Aegilops species and determination of chromosomal localizations of microsatellites in the S genome," Genome, vol. 48, no. 6, pp. 959-970, 2005.

[14] G. Ablett, H. Hill, and R. J. Henry, "Sequence polymorphism discovery in wheat microsatellite flanking regions using pyrophosphate sequencing," Molecular Breeding, vol. 17, no. 3, pp. 281-289, 2006.

[15] L. Ayala-Navarrete, E. Tourton, A. A. Mechanicos, and P. J. Larkin, "Comparison of Thinopyrum intermedium derivatives carrying barley yellow dwarf virus resistance in wheat," Genome, vol. 52, no. 6, pp. 537-546, 2009.

[16] M.-S. You, B.-Y. Li, Z.-H. Tang, Z.-H. Tang, S.-B. Liu, and G.T. Liu, "Development of specific SSR marker for Ee genome of Thinopyrum spp. using wheat microsatellites," Chinese Journal of Agricultural Biotechnology, vol. 1, pp. 143-148, 2004.

[17] D. J. Mullan, A. Platteter, N. L. Teakle et al., "EST-derived SSR markers from defined regions of the wheat genome to identify Lophopyrum elongatum specific loci," Genome, vol. 48, no. 5, pp. 811-822, 2005.

[18] Wheat Applied Genomics, http://maswheat.ucdavis.edu/. 

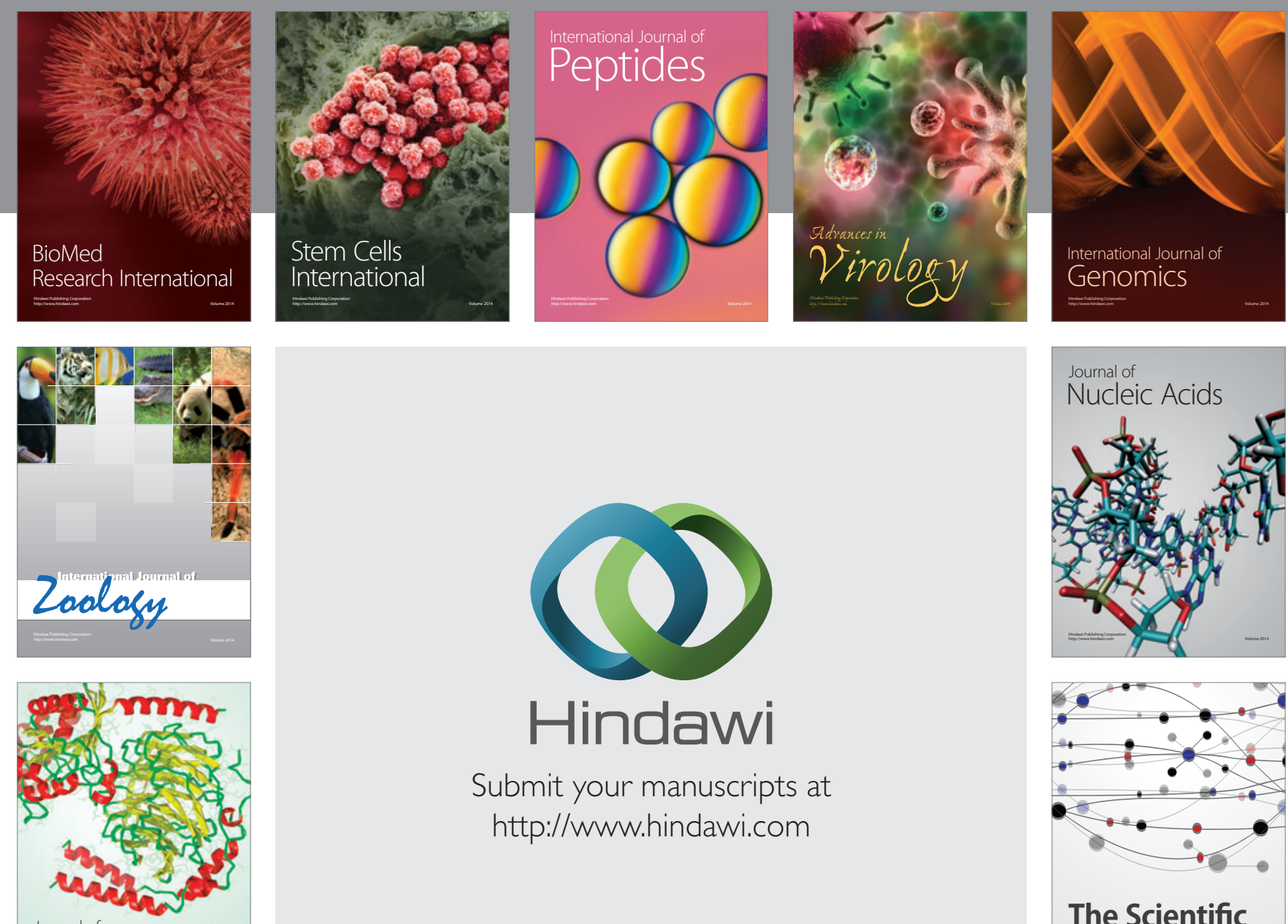

Submit your manuscripts at

http://www.hindawi.com

Journal of
Signal Transduction
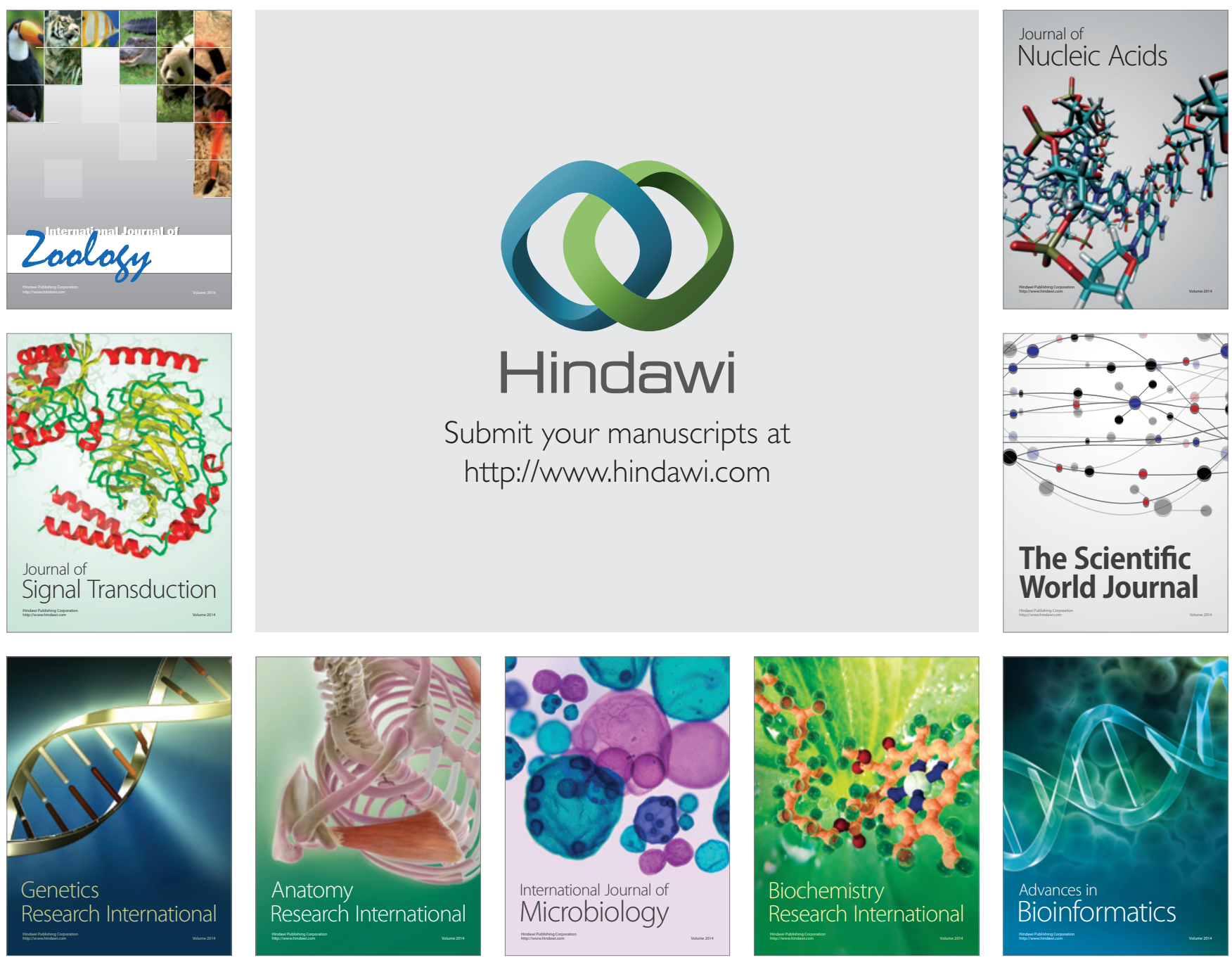

The Scientific World Journal
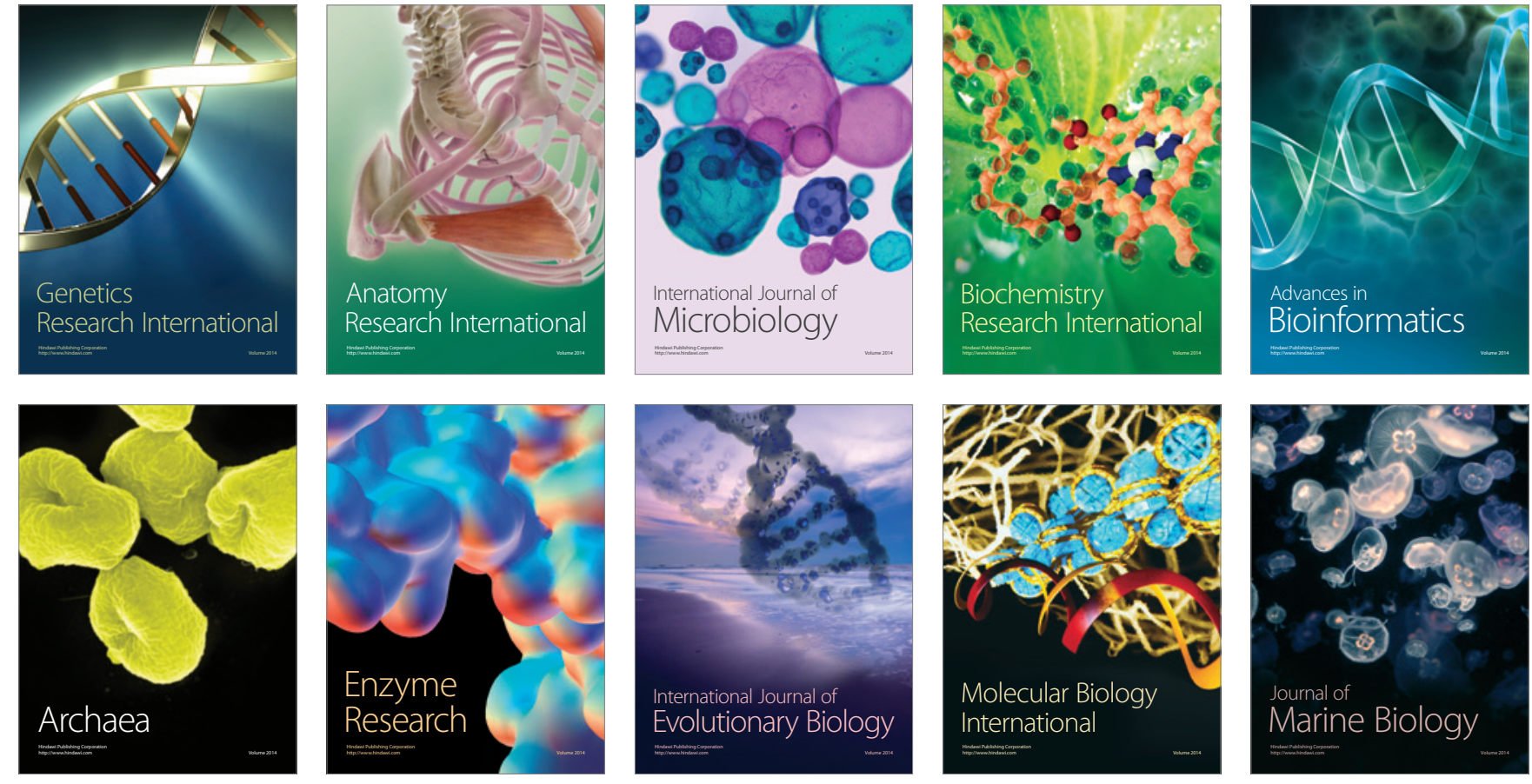